Chapter 4

\title{
New Insights into the Optical Coherence Tomography - Assessement and Follow-Up of Age-Related Macular Degeneration
}

\author{
Simona-Delia Țălu \\ Additional information is available at the end of the chapter \\ http://dx.doi.org/10.5772/53357
}

\section{Introduction}

Optical coherence tomography (OCT) is a relatively new noninvasive optical imaging method that has revolutionized the way we see the retina. It uses near-infrared light in order to deliver high-resolution cross-sectional images of the macula that are very similar to the histopathological specimens. However, the OCT images are not the direct depiction of the anatomical structures, but they represent the consequence of the optical properties of the tissues being scanned [1]. The retinal microarchitecture can be visualized as cross-sectional or tomographic volumetric data. The OCT has been used increasingly over the past several years to diagnose and monitor a variety of retinal diseases that affect the macula. Age - related Macular Degeneration (AMD) is one of the retinal diseases that benefited the most from the development of OCT techniques. In the exudative form of the disease, OCT identifies intraretinal fluid and outlines the choroidal neovascularization. However, it is not able to resolve the internal structure of the fibro-vascular membranes, as it cannot distinguish very clearly between their various components: new vessels, fibrous tissue, blood, dense exudates. OCT is extremely useful in the management and monitoring of wet AMD: it allows early diagnosis and helps in the decision-making process of retreatment with anti-VEGF agents [2]. OCT became an indispensable tool in the diagnosis of neovascular recurrences by identifying fluid under or in the retina [3]. Newer spectral domain OCT devices permit the detailed description of drusens in early AMD that allows the refined phenotyping of the disease [2]. By allowing detailed description of various lesions in AMD, OCT is bringing significant contributions for progressing in the understanding of AMD pathogenesis. 


\section{Problem statement}

A variety of retinal diseases such as AMD, central serous chorio-retinopathy, macular hole, vitreo-macular interface syndrome and diabetic maculopathy have taken advantage from the introduction of OCT in the clinical practice. Among these, AMD is the ocular condition that benefited the most from the enormous advantages offered by OCT imaging techniques, in terms of diagnosis, response to treatment and monitoring. Future progress in OCT techniques has already brought and is expected to bring new insights in understanding the pathophysiology of this potentially blinding disease. With the aim of defining the role of OCT in the assessment and follow up of AMD, the theoretical principles at the foundation of this retinal imaging technique are outlined, making a clear distinction between TD-OCT and SDOCT methods. The advantages of SD-OCT over TD-OCT methods are revealed. The role of OCT in AMD management is emphasized by the description of typical OCT aspects in various AMD lesions: macular edema, CNV membranes, occult and classic choroidal neovascularization, pigment epithelial detachments (PED), external retinal cysts, vitreo-macular adhesions. OCT has a crucial role in monitoring AMD and establishing the indication of treatment with anti-VEGF intravitreal injections in the wet form of the disease. The place of OCT in the evaluation of AMD patients is completed by its comparative presentation with retinal biomicroscopy, fluorescein and indocyanine green angiography. The impact of OCT in AMD diagnosis and monitoring is illustrated with examples of various aspects that AMD can display, both with a TD-OCT device (Stratus OCT) and a SD-OCT one (Cirrus OCT). Future directions in OCT techniques development close the current presentation.

\section{Theoretical considerations on OCT examination techniques}

\subsection{History}

The concept of optical coherence tomography (OCT) was developed at Massachusetts Institute of Technology in the early 1990s and the first commercial version of OCT was made available by Carl Zeiss (Jena, Germany) in 1996 [4]. The first applications of OCT were to provide quantitative and qualitative information about the peripapillary area of the retina and the coronary artery [5]. The first publication on time-domain OCT belongs to Huang and coworkers in 1991 [5]. Hee et al. published the first data on the quantitative evaluation of the macular edema in 1995 [6]. Since its introduction, OCT evolved into a powerful examination tool for patients with retinal diseases [7].

\subsection{Optical tomography versus ultrasound}

For many years, the cross-sectional imaging of the eye has been possible only with the help of ultrasounds which allowed spatial resoultions of $150 \mu \mathrm{m}$. The development of new techniques was based on the use of higher frequency waves that created the possibility to obtain image resolutions of $20 \mu \mathrm{m}$. However, the strong atenuation in the biological tissues limits its use to the anterior structures of the eye [8]. The principles of the two imaging methods 
(OCT and ultrasounds) are similar, but OCT uses light instead of sound. The primary difference between ultrasonic and optical imaging derivates from the different speed of sound and light. The light propagates nearly a million of times faster than sound, which allows the obtaining of measurements resolutions in the range of $10 \mu \mathrm{m}$ or less at the posterior pole of the eye. Also, the use of light makes the examination more comfortable for the patient, as there is no need for physical contact with the examined eye [8].

\subsection{OCT Instrumentation for retinal imaging}

The device operates as a fundus camera: a condensing lens of +78 dioptres is used so that the retina could be imaged in the same plane with the instrument. The magnification of the retinal image is determined by two factors: the refractive power of the condensing lens and the magnification of the ocular. At the lowest magnification, the typical field of view is $30^{\circ}$. If the visual acuity of the eye to be examined is very low and therefore the patient has fixation problems, a guiding light can be placed in front of the fellow eye, in order to stabilize the eyes during image acquisition. With the first generation OCT devices, pupil dilation was recommended with the aim of obtaining high quality images, but the latest generation OCT machines deliver good quality pictures with a 3-mm diameter pupil. The OCT technology can be limited by various conditions of the eye: lens or vitreal opacities, subretinal haemorrhages, lack of foveal fixation, nystagmus [8].

\subsection{Principle of OCT}

OCT is based on the interferometry that uses a low-coherence light in order to measure the difference between the reflected light waves from the examined tissue and that from a reference path [9]. The light reflected from the retinal structures interferes with the light of the reference beam. The detection of echoes resulting from this interference is measuring the light echoes versus depth $[9,10]$.

Axial resolution. Axial resolution is given by the bandwdith of the light source and the level of coherence that depends upon the central wavelength [10]. The light from a superluminiscent diode has long coherence that generates images with poor axial resolution. With shortcoherence light, interference is possible over short distances and therefore high axial resolution is possible [10].

Transverse resolution. The transverse resolution is determined by the size of the light spot that can be focused on the retina. The best structural resolution is obtained when the light is focused on the tissue to be examined. The absorbtion of central light by the tissue of interest must also be considered [10].

\subsection{Time Domain (TD-OCT) versus Spectral Domain (SD-OCT)}

OCT is applied by two main methods: TD-OCT and SD-OCT.

TD-OCT produces two-dimensional images of the sample internal structure. An A-scan represents a reflectivity profile in depth which is gradually built up over time by moving a mir- 
ror in the reference arm of the interferometer. A B-scan depicts a cross-section image meaning a lateral $x$ depth map which is generated by collecting many A-scans [4].

SD-OCT can be implemented in two formats, Fourier domain (FD-OCT) and swept source (SS-OCT). With SD-OCT units, all the A-scans in the reflected light are acquired at a given point in tissue. The moving mirror is not needed in order to obtain complete A-scans which allows the acquisition of the images about 60 times faster than with TD-OCT. The detection and monitoring of retinal diseases is improved with SD-OCT units because they have ultra high-speed scan rate, superior axial and lateral resolution, cross-sectional (2D) scan, 3D raster scanning and a higher imaging sensitivity than the traditional TD-OCT units. The SDOCT software is much improved compared with traditional TD-OCT and the great number of scans done per unit of time creates the conditions for the SD-OCT systems to generate 3D reconstructions which can be further manipulated with the aim to demonstrate subtle pathology not evident with conventional 2D images [4].

Briefly, the advantages of SD-OCT over TD-OCT are: significant improvement of the image axial resolution, decreased acquisition times, reduction of motion artifacts, increased area of retinal sampling and the possibility to create topographic maps by the three-dimensional evaluation of tissues [11,12].

Table 1 summarizes the main differences between TD-OCT and SD-OCT.

\begin{tabular}{|c|c|c|}
\hline Property & TD-OCT & SD-OCT \\
\hline Principle & Low coherence interferometry & Fourier transformation \\
\hline Modality of acquisition & $\begin{array}{l}\text { An interferometer measures } \\
\text { sequentially the echo delay time of light } \\
\text { that is reflected by the retinal } \\
\text { microstructures }\end{array}$ & $\begin{array}{c}\text { A spectrometer evaluates } \\
\text { simultaneously the light reflection by } \\
\text { the retinal microstructures }\end{array}$ \\
\hline Modality of sampling & It samples one point at the time & It samples all the points simultaneously \\
\hline Image acquisition time & $1-2$ seconds & 60 times faster \\
\hline Sampled area & $\begin{array}{l}6 \text { radial scans are performed, } 20 \mu \text { wide } \\
\text { and } 6 \mathrm{~mm} \text { long (the area between the } 6 \\
\text { scans is not imaged) }\end{array}$ & $\begin{array}{l}\text { In a 6-mm diameter area, } 65.000 \text { scans } \\
\text { are performed, without excluding areas; } \\
\quad 128-200 \text { scans over the same area }\end{array}$ \\
\hline Rate of acquisition & $\begin{array}{l}\text { Cross-section images of the retina are } \\
\text { obtained every } 1.6 \text { seconds: } 400 \text { scans/ } \\
\text { second }\end{array}$ & $25.000-52.000$ scans/second \\
\hline Presentation of the results & $\begin{array}{l}\text { Two-dimensional images of the sample } \\
\text { internal structure }\end{array}$ & $3 \mathrm{D}$ reconstruction possible \\
\hline Image axial resolution & $10-15 \mu \mathrm{m}$ & 3-7 $\mu \mathrm{m}$ \\
\hline
\end{tabular}

Table 1. The main differences between the properties of TD-OCT and SD-OCT 


\section{TD-OCT}

\subsection{Principle of TD-OCT}

The origin of TD-OCT imaging technique is found in the processes of absorbtion and dispersion of light traversing tissues [13]. The creation of an image with the TD-OCT technique is based on the principle of low coherence interferometry. The source of light is represented by a superluminiscent diode that emits a radiation with the wavelength of 830-840 $\mathrm{nm}$. This emission is split in two arms by an optical beam splitter functioning as interferometer: half the beam is reflected from the reference mirror and is named the reference beam and half of it is directed to the target tissue and is named the detection beam. The comparison of the tissue-reflected beam with the beam coming from the reference mirror measures the time delay between these two beams [13]. In order to understand the system operating, the corpuscular theory of light must be applied: the beam is made up of short pulses of light. The pulse of light reflected from the reference mirror and the pulse of light coming from the analyzed tissue within the eye will coincide only if they both arrive at the same time, producing the phenomenon called light interference. For the light interference to occur, the distance traveled by the two above mentioned beams must be equal. The interference is measured by a light-sensitive detector and it is translated into OCT image on the screen [8]. This method allowed to obtain cross-section images of the retina every 1.6 seconds (400 A-scans per second) [14]. TD-OCT has limits represented by: long acquisition times, limited image sampling (with the risk of overlooking small macular lesions), limited resolution by motion artifacts and patient blinking [14].

\subsection{Tomographic imaging and volumetry - Interpretation of TD-OCT images}

The light source moves across the retina and the optical reflection and backscatter from the retinal structures are detected. Successive longitudinal measurements at transversal sequential points are performed. This technique generates a two-dimensional image and a crosssectional map displayed in false colours. Each colour is given a certain degree of reflectivity. White and red colours corespnd to highly reflective tissues, whereas black and blue represent low reflectivity structures. Green is given an intermediate reflectivity. Examples of hyperreflective tissues are: fibrosis, haemorrhages, infiltrates [8]. The retinal layers are displayed on the linear scans and the retinal thickness can be measured taking as references the vitreo-retinal interface and the retinal pigmented epithelium, given their different reflectivity. By using 6 radial scans 30 degrees apart, a surface map can be obtained, in which white and red represent high volume structures (for example, macular edema) and black and blue correspond to thinned retinal areas [8].

\subsection{Image resolution}

The most important parameter that determines OCT image resolution is the coherence length of the light source. For the commercially available TD-OCT system, image axial resolution is in the range of $10-15 \mu \mathrm{m}$. The penetration through transparent optical media is excellent, but through a thick haemorrhage is less than $100 \mu \mathrm{m}$ [8]. 


\subsection{Image processing and correction for eye motions}

Image acquisition takes about 1-2 seconds. Taking into account that the image resolution is extremely high, the correction for eye motions is very important to avoid the obtaining of blurred images. As consequence, image processing techniques had to be developed [8].

\section{SD-OCT}

\subsection{Principle of SD-OCT}

The development of the SD-OCT technique is originating in the Fourier mathematical equation (1807). The french mathematician Joseph Fourier described the decomposition of a periodic function into a sum of simple sinusoidal-based oscillating functions. The practical effect of this abstract statement is the possibility to measure simultaneously all echoes of light, in contrast to TD-OCT where the echoes of light are measured sequentially by moving a mirror in front of the reference beam. The SD-OCT devices use a central wavelength of $800-850 \mathrm{~nm}$, a stationary reference arm, a high speed spectrometer that analyses simultaneously all the frequencies and a charged-coupled device (CCD) line-scan camera. The mechanical scanning is not needed in order to detect light echoes simultaneously. As consequence, the aquisition speed increases to 25,000-52,000 A-scan/second and the amount of data that can be obtained during one session was improved significantly [15]. The axial resolution is of 3-7 $\mu \mathrm{m}$ (as compared to $10-15 \mu \mathrm{m}$ with TD-OCT devices), significantly improving the signalto-noise ratio. Therefore, the detection of individual retinal layers and lesions components became possible [16].

\subsection{Clinical impact of SD-OCT}

The practical impact of the improvement in axial image resolution is the early detection of small cystic changes associated with the wet form of AMD. The early diagnosis is very important for the early treatment and the better preservation of the visual function. Given the possibility to get images simultaneously in various planes, the 3D reconstruction is possible with SD-OCT, allowing the obtaining of hundreds of high-resolution images per second and the accurate measurement of the macula (total volume) in various conditions: edema, fluid, drusen, CNV. The reduction of the examination time considerably decreases the artifacts related to eye movements and poor fixation of the low vision patients [17]. Another significant advantage of SD-OCT is represented by the increased retinal scan coverage. The SD-OCT images have proven to be clearer and with higher quality as compared to the ones obtained by the successive TD-OCT systems (OCT1, OCT3, stratus). The SD-OCT systems are continuously improving, by adding complementary functions: fundus photography, angiography, microperimetry. The ultra-high resolution images obtained by SD-OCT allow a better differentiation between the retinal and subretinal layers [4]. 


\subsection{Commercially available SD-OCT devices}

SD-OCT has superior depth resolution as compared to TD-OCT. Currently, the axial resolution varies from $3-7 \mu \mathrm{m}$, depending on the SD-OCT model [18]. Several SD-OCT instruments are available at the current moment: Cirrus HD-OCT (Carl Zeiss Meditec), RTVueFourier DomainOCT (Optovue), Copernicus OCT (Reichert/-Optopol Technology), Spectral OCT/SLO (Opko/Oti), Spectralis HRA+OCT (Heidelberg Engineering), Topcon 3D OCT-1000 (Topcon) and RS-3000 Retiscan (Nidek). All the above mentioned instruments provide high quality images and offer the possibility of tridimensional reconstruction of the macula [13]. The main technical characteristics and differences between the commercially available SD-OCT devices are presented in table 2 [15].

\begin{tabular}{lcc}
\hline Device (Company) & Axial resolution $(\boldsymbol{\mu m})$ & A-scans/second \\
\hline Cirrus HD-OCT (Carl Zeiss Meditec) & 5 & 27000 \\
\hline Spectralis (Heidelberg Engineering) & 7 & 40000 \\
\hline RTVue-100 (Optovue) & 5 & 26000 \\
\hline 3D-OCT 1000 & 6 & 18000 \\
3D-OCT 2000 (Topcon) & 5 & 27000 \\
\hline Spectral OCT/SLO (OPKO/OTI) & 6 & 25000 \\
\hline SOCT Copernicus (Optopol) & 3 & 50000 \\
\hline SOCT Copernicus HR (Canon/Optopol, Inc) & 4 & 20000 \\
\hline SDOCT (Bioptigen) & 7 & 53000 \\
\hline Retinascan RS-3000 (Nidek) & 4 & \\
\hline
\end{tabular}

Table 2. Commercially available SD-OCT devices

\section{Application area of OCT in AMD}

\subsection{Overview}

Most cases of neovascular AMD are complicated by intraretinal fluid accumulation and RPE detachments. For many years, the therapeutic decision in neovascular AMD was based on the results of fundus biomicroscopy, fluorescein and indocyanine green angiography. In this context, the posttherapeutical evolution in many $\mathrm{CNV}$ membranes remained unsatisfactory. OCT technology offers subtle imaging of CNV membranes that appear as hyperreflective bands on OCT. Frequently, the identification of CNV depends on the reflectivity of the adjacent structures and on the CNV localization related to it. OCT is more sensitive than biomicroscopy in identifying retinal edema and small neurosensory and RPE detachments. The OCT relationship with fundus biomicroscopy and fluorescein angiography (FA) in AMD patients is summarized in table 3 [8]. 
OCT is more reliable than biomicroscopy in assessing the macular thickness and small neurosensory and RPE detachments.

OCT is more reliable than FA in identifying the intraretinal and subretinal fluid, as on FA the fluid in the inner retina can mask the fluid in the outer retina.

OCT is less precise in evaluating the geographic extent of fluid within the macula, as compared to FA.

Due to the cross-sectional imaging, OCT allows the localization of a CNV in relationship with the RPE and the neurosensory retina.

OCT is superior to FA in identifying CNV membranes that are obscured by pooling of dye or by tiny retinal haemorrhages.

Cystoid macular edema is strongly associated with the classic CNV.

$\mathrm{OCT}$ is very useful in AMD monitoring and decision-making after treatment: if fluid persists, re-treatment is indicated.

Table 3. Place of OCT in the ophthalmic evaluation of the AMD patients - summary for the clinician

\subsection{OCT measurement of macular thickness}

In $\mathrm{AMD}$, the most important clinical parameter is the central macular thickness. OCT is the most precise method to measure retinal thickness in vivo. However, OCT measurements cannot be correlated exactly to the histopathologic ones, because OCT signals are directly determined by the optical properties of tissues. Therefore, structures that stain strongly on the histopathological specimens do not necessarily appear as intense OCT signals. It has been demonstrated that there are differences in the retinal thickness measurements between OCT models, possibly explained by the higher axial and transverse resolution of the newer devices. For instance, a comparison was made between Cirrus and Stratus OCT devices in measuring the macular thickness [19]. Firstly, the definition of the retinal boundaries used by the automated segmentation algorithms differs between devices [19]. According to the manufacturer, the Stratus OCT program measures between the nerve fiber layer and the inner boundary of the RPE complex, though it has been reported that Stratus OCT has two outer reference lines: one at the junction between the inner/outer segment of the photoreceptor cells and the other at the inner boundary of the RPE. The Cirrus OCT program measures the retinal thickness between the nerve fiber layer and the outer band of the RPE. In consequence, the Cirrus outer reference band is deeper than the first mentioned Stratus external band and is closer to the second mentioned one. The correlation of thickness measurements between the two devices is modest, as the Cirrus OCT provides greater measurement depth. The practical implications are targeted towards the clinical practice (the patients participating in clinical trials may not interchange between Stratus and Cirrus OCT systems, patients transiting from one practice to another should have the tests done with the same OCT model) and the manufacturer (upgradation of Stratus OCT software). Secondly and more importantly, the TD-OCT calculates the retinal thickness based on 6 radial line scans, whereas SD-OCT uses data of 3D scan with 128-200 scans over the same area [19]. Thus, TD-OCT evaluates a small macular area, whereas SD-OCT images almost the entire macular region. 


\subsection{OCT imaging of CNV}

In neovascular AMD, the retina is invaded by new vessels originating in the choroidal vessels. According to the relationship of these new vessels with the retinal architecture, wet AMD presents in two forms: classic CNV and occult CNV. The histopathological studies proved that in classic CNV the new vessels penetrate Bruch's membrane, the RPE and the neural retina, whereas in occult CNV they are located between the RPE and Bruch's membrane, with subsequent loss of RPE barrier function. In classic CNV the RPE is elevated by exudates, blood and lipids located in the subretinal space and having the origin in the new vessels. The occult $\mathrm{CNV}$ can be further divided in two forms: with or without serous pigment epithelial detachment [20] (Table 4). Cystoid macular edema was statistically strongly correlated with the classic form of choroidal neovascularization, whereas the absence of cystoid macular edema was statistically strongly correlated with the occult choroidal neovascular membranes.

\begin{tabular}{lll}
\hline CNV type & Location of the new vessels & Clinical findings \\
\hline Classic & $\begin{array}{l}\text { Penetrate Bruch's membrane, RPE, } \\
\text { neural retina }\end{array}$ & RPE elevated by blood, exudates, lipids \\
\hline Occult & Between Bruch's membrane and RPE & With PED \\
\cline { 2 - 2 } & & Without PED \\
\hline
\end{tabular}

Table 4. Summary of CNV types - correlation of the histopathological and clinical findings

Occult CNV were described initially on fluorescein angiography (FA) and then on indocyanine green angiography (ICGA) that detected them more accurately, mainly if Scanning Laser Ophthalmoscopy (SLO) was also used. In the recent years, OCT proved its usefulness in defining the features of occult CNV. The main OCT finding that defines occult CNV is the RPE elevation that is separated from the underlying choroidal plane. The cavity formed by the RPE elevation is most frequently fibrovascular and therefore coloured in green, but it can also be serous and then appears optically empty. The RPE elevation is poorly delineated and its extent is variable according to the stage of the lesion. In occult CNV, the RPE elevation can be associated with other OCT signs: modifications of the RPE band (hyperreflectivity, fragmentations, thickening, thinning), subretinal and intraretinal accumulation of fluid, vitreomacular traction syndrome [21].

\subsection{OCT in the evaluation of the therapeutical response to photodynamic therapy in wet AMD}

OCT helped to better describe the response to treatment in wet AMD. For instance, the response to photodynamic therapy takes place in 5 stages: in the first stage, there is a mild fluid accumulation that corresponds to an acute inflammatory reaction after PDT. Approximately 4 weeks after the first treatment, if there is fluid accumulation, retreatment is suggested. Typically, the fluid accumulates in the subretinal space, causing the 
detachment of the neurosensory retina. Cystoid macular edema is evident in the penultimate stage, approximately 5 months after PDT and it is associated with important subretinal fibrosis on OCT. In the last stage, there is complete resolution of retinal fluid, concomitant with subretinal fibrosis and retinal atrophy. In conclusion, OCT has a significant impact on the therapeutic approach in AMD: the presence of subretinal fluid proves an active CNV and further therapy is indicated, the presence of fibrosis is associated with cystoid macular edema that has no benefit from therapy as compared to its natural evolution [20].

\subsection{OCT imaging of Pigment Epithelial Detachments (PEDs)}

In the context of the chorio-retinal diseases, AMD is the condition in which PEDs are the most frequently identified. As hypothesized by Gass, PEDs occur in AMD by two mechanisms: serous exudation from hyperpermeable vessels of the choriocapillaris through an intact Bruch's membrane or neovascular ingrowth with subsequent exudation from the new vessels directly in the subretinal space [22]. Using a TD-OCT device, which was not able to visualize within the PED, Coscas was the first to observe that the presence of a layer of tissue behind the RPE in PEDs is associated with occult CNV. The new devices allow the description of the PEDs content: the dark regions within them are not attributable to the low sensitivity of the imaging technique, but to the hyporeflectivity of the region itself [22]. Before the OCT era, PEDs were assessed manually or automatically according to their areas. However, PEDs can have similar areas but very different volumes, their three-dimensional evaluation being therefore very important. Cirrus SD-OCT system can be used in order to measure the area and volume of PEDs with the help of an algorithm similar to the one reported for the measurement of drusen and that proved to be highly reproducible [23]. The PEDs usually indicate the presence of $\mathrm{CNV}$ and are associated with poorer visual outcome. Therefore, their identification carries a prognostic value $[22,23]$.

\subsection{OCT imaging of vitreomacular adhesions}

Although AMD involves primarily the outer retinal layers, is was suggested by several authors that vitreous may play a role in AMD pathogenesis and/or progression [24]. There is clinical evidence that vitreomacular adhesions may play a role in AMD development. It was proved that the prevalence of PVD was significantly lower in patients with AMD as compared with healthy subjects [25]. In patients with partial PVD, the region of vitreomacular adhesion corresponded to the area of subretinal neovascularization [26]. Also, the incidence of persistent vitreomacular adhesion was significantly higher in eyes with wet AMD as compared to those with dry AMD and controls. Vitreous was also demonstrated to be important in inducing PEDs. If future prospective studies will confirm these findings, it seems appropriate to use OCT in order to assess the vitreomacular relationship in AMD patients. Subsequently, the OCT findings could guide the prevention of wet AMD, by establishing the indication for vitrectomy or pharmacologic vitreolysis [24,25,26]. 


\subsection{Choroidal thickness measurements}

Choroid is a very important structure for AMD, because abnormalities at the level of the choroidal circulation seem to be the most significant factor involved in the development of this disease. The use of the newest OCT software enables the measurement of choroidal thickness, but only few studies evaluated it in AMD. So far, it has been proved that the choroidal thickness is variable among patients with AMD. In the group of patients with thicker than normal choroid it is difficult to include them in one of the three following cathegories: a variant of normal, a subset of AMD or a different entity. An overall concluison is that the patients with wet AMD displayed thinner choroids than the ones with dry AMD. These findings postulate that the continuing thinning of the choroid might be a factor indicating the risk of progression towards wet AMD. Therefore, the OCT evaluation of the choroidal thickness could be a valuable tool in identifying the patients at risk to develop the wet form of AMD, thus allowing the early diagnosis and therapy. The thinner than normal choroid induces ischemia at the level of RPE, stimulating the production of VEGF and subsequent proliferation of new vessels. Until now, OCT studies could not establish a correlation between the choroidal thickness and parameters with practical importance: number of intravitreal anti-VEGF injections, duration of the disease, visual acuity [27].

\subsection{External retinal cysts}

The new OCT imaging techniques allowed to identify the external retinal cysts that differ from the ones in cystoid macular edema and therefore to avoid the useless treatment. They are not perfectly understood yet, but the intravitreal anti-VEGF injections don't seem to influence their evolution. The origin of the external retinal cysts is explained by two theories: inflammatory and mechanical. The inflammatory theory postulates that following photoreceptor and pigment epithelial cells degeneration induced by intravitreal injections of antiVEGF agents, specific macrophages appear in order to digest the cellular debris. It seems that the external retinal cysts are giant activated macrophages. This theory is sustained by the localization of these cysts in areas of high concentrations of oxidized lipoproteins: in front of the ancient fibro-vascular lesions and of the extended regions of retinal and pigment epithelial atrophy. In accordance with the mechanical theory, the external retinal cysts are rolled photoreceptor cells. During the process of retinal degeneration, the tight junctions between the external segments of the photoreceptor cells disappear and they subsequently form tube-like structures in the external retina. OCT findings exclude the mechanical theory, as the tube-like structures were never identified in AMD, whereas the segmentation OCT techniques proved that the "tubes" correspond to plies of the pigment epithelium and not to rolled photoreceptors. The OCT identification of the external retinal cysts carry an unfavorable prognosis as they are associated with ancient degenerative lesions and with the disappearance of the external retinal layers [3].

\subsection{OCT in dry AMD}

In dry AMD there is atrophy of the choroid with subsequent degeneration of RPE and involution of the photoreceptor and external retinal layers. However, even if the RPE becomes 
atrophic, it preserves the barrier function, keeping the macular region dry [20]. The current OCT systems allow the identification of drusen. The small and intermediary size ones appear as discrete elevations of the RPE with variable reflectivity, according to the underlying material. In the large drusen (or so-called drusenoid PEDs) the RPE displays greater, often dome-shaped elevations separated from the Bruch membrane by a hypo or medium reflective material. On OCT images drusen are often accompanied by modifications in the neurosensory retina, translated by the thinning of the outer nuclear layer and disruption at the level of the external limiting membrane and of the inner segment (IS)-outer segment (OS) junctions. These observations are in agreement with the histopathological ones that demonstrated the loss of photoreceptor cells in patients with drusen [28]. Geographic atrophy (GA) appears on OCT as sharply demarcated areas of choroidal hyperreflectivity due to the loss of RPE. If retinal atrophy is associated, thinning or loss of the outer nuclear layer, absence of the external limitting membrane and of the IS-OS junctions are seen. In the areas of GA, islands of preserved retina may be identified, as well as regressing drusenoid materials appearing as hyperreflective plaques at the RPE band. In the GA in the inner nuclear layer may be identified small cystic-like spaces in the absence of any macular edema [28]. The evaluation of GA in terms of size, location and rate of progression is crucial in assessing the visual prognosis of the patients. An important finding that identifies the rapidly progressive cases is the autofluorescence surrounding the GA areas. OCT also revealed in the junctional zones of GA that the outer plexiform layer approaches the Bruch membrane suggesting that photoreceptor loss extends beyond the limits of the lesion. OCT reveals dynamic changes in the junctional zones of GA: pigment migration, variation in drusen height. In conclusion, OCT examination in dry AMD is important in two main directions: it provides insights in the disease pathogenesis and it allows the prediction of visual outcome [28].

\subsection{OCT versus angiography in AMD}

The advantages of OCT over fluorescein angiography (FA) are represented by: better structural identification of $\mathrm{CNV}$, identification of a $\mathrm{CNV}$ masked by the pooling of dye or by thin haemorrhages. On FA, in order to suspect the retinal edema the source of leakage has to be active, whereas on OCT even the minimal edema can be objectivized, no matter if the source of leakage is active or not. Cystoid macular edema in exudative AMD is difficult to be vizualized on FA because of the leakage that obscures the accummulation of fluid in the inner retina. Based on the OCT findings, the prevalence of cystoid macular edema in the cases of subfoveal CNV due to AMD in a retrospective study was estimated to be arround $46 \%$. It has been demonstrated that during all the phases the ICGA substantially underestimated the size of the neovascular complex in comparison to SD-OCT. This could be explained by the high molecular weight and affinity of indocyanine green for the albumin molecules that prevented its even distribution through the entire lesion. Well defined hyperfluorescence during the early phase of FA defining the neovascular complex also underestimated the size of the lesion measured with SD-OCT. On the other hand, despite the SD-OCT capabilities of delivering high resolution images, the components of a fibrovascular complex may represent other subretinal material, particularly of inflammatory origin. Another important observation refers to the extent of leakage during the late phase of FA that did not reach the 
extension of subretinal or intraretinal fluid objectivized on SD-OCT or even TD-OCT images. It was postulated that minimal fluid on the OCT images might not represent leakage from a newly formed CNV, but failure of liquid resolution by the PRE. Therefore, some authors recommend retreatment by intravitreal anti-VEGF injections only if the presence of fluid on SD-OCT is associated with leakage on FA. However, since SD-OCT is more reliable in detecting extravasated fluid, retreatment based on SD-OCT parameters should be more effective, especially that the pharmacologic therapies act by reducing leakage rather than by an antiproliferative effect [29]. The OCT technology allows new diagnostic criteria for AMD and the decision-making process and AMD monitoring without the need to subject the patient to fluorescein angiography [8]. The ultrahigh-resolution OCT uses a titanium-sapphire laser light source that delivers image resolutions of $3 \mu \mathrm{m}$ [8]. This is particularly useful in neovascular AMD for the precise evaluation of sub-RPE and sub-foveal choroidal neovascular membranes [8]. On OCT basis, Hee et al. proposed a simple classiffcation scheme of exudative AMD into three categories which does not always correlate with the FA aspects: welldefined $\mathrm{CNV}$, poorly-defined $\mathrm{CNV}$, fibrovascular pigment epithelial detachment. In poorlydefined $\mathrm{CNV}$, the choroidal reflectivity is diffusely increased and is associated with intraretinal or subretinal fluid accummulation that appears hyporeflective. The presence of the fluid or the small disruptions at the level of the RPE or choriocapillaris differentiate the poorly-defined CNV from the RPE atrophy that is translated on OCT as increased choroidal reflectivity as well. The introduction of OCT in the clinical practice was followed by the modification of the classification schemes based on FA. Thus well-defined CNVs or fibrovascular pigment epithelium detachments appear as precisely demarcated boundaries on OCT, whereas on FA sometimes they were classified as occult choroidal neovascular membranes. Poorly-defined CNVs correspond to angiographically occult CNVs in most instances. The emerging conclusion of these practical observations is that OCT provides anatomical details that are not evident on FA. The most important consequence is the optimization at the therapeutic level, as the approaches in angiographically classic and occult CNVs are different $[8,29]$.

\section{Stratus OCT in clinical practice}

We used the Stratus OCT device (Carl Zeiss Meditech) with the fast macular map scan protocol that consists in 6 radial scans oriented 30 degrees from one another, each having a 2$\mathrm{mm}$ axial depth and 6-mm transverse length. Each image had $10 \mu \mathrm{m}$ axial and $20 \mu \mathrm{m}$ transverse resolutions in tissue with a maximum scan velocity of 400 axial scans per second.

Figures 1a and $\mathrm{b}$ depict the case of a patient with wet AMD with significantly increased macular thickness (central foveal thickness of $427 \mu \mathrm{m}$ ). Macular edema is provoqued not only by the CNV visible on the cross-sectional images of the retina, but also by an increased vitreo-macular adhesion. The relatively long evolution of the disease is suggested by the modifications in the internal retinal layers, with hyporeflective cysts within their structure. The OCT aspect confirms the theory that the maximal vitreo-macular adhesion is located in front of the subretinal neovascular membrane. 

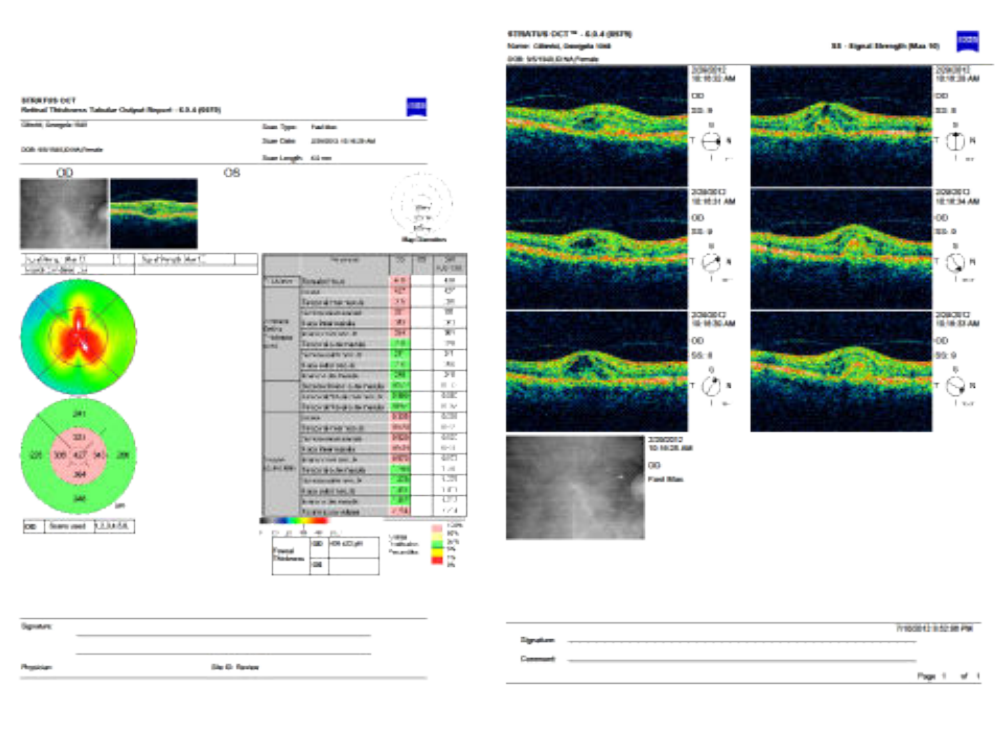

Figure 1. $a$ and b Retinal thickness: increased macular thickness due to CNV but also to vitreo-macular adhesion (courtesy of dr. H. Demea, Review Centre, Cluj-Napoca, Romania)

In figures $2 \mathrm{a}$ and $\mathrm{b}$ a bilateral AMD case is illustrated. In both eyes the RPE is elevated by a hyperreflective structure (CNV) and the macular thickness is increased. After 2 intravitreal injections with Bevacizumab (Avastin) the RPE elevation and the macular thickness decreased, as shown in figures $3 a$ and $b$.

Figure 4a shows a macular edema in the left eye of a patient with AMD and figure 4b displays its aspect 20 months after periodic intravitreal injections with Bevacizumab (Avastin). Improvement is obvious, both regarding the central macular thickness and the cross-sectional aspect of the macula. Decision whether to treat or not was based on the OCT aspects, taken on a monthly basis.

The Stratus OCT images in figure 5a and 5b prove an elevation of the RPE in the right eye with moderately increase of central macular thickness $(279 \mu \mathrm{m})$. One month after intravitreal injection with Bevacizumab (Avastin) the central macular thickness decreased to 246 $\mu \mathrm{m}$, as shown is figure $6 \mathrm{a}$ and $6 \mathrm{~b}$.

Figures 7a and 7bare illustrating the Stratus OCT aspects of the left eye of a patient with AMD: there is a marked increase in the central macular thickness $(684 \mu \mathrm{m})$ and the retinal structure appears disorganized. One year after periodic intravitreal injections with Bevacizumab (Avastin) the central macula appears considerably thinner $(380 \mu \mathrm{m})$ and the retinal layers much better arranged, as proved in figures $8 \mathrm{a}$ and $8 \mathrm{~b}$.However, in the internal retinal layers cystic structures appear, suggesting cystoid macular edema. As in case illustrated in figure 4 , the decision of treatment was taken exclusively according to the OCT aspects. 

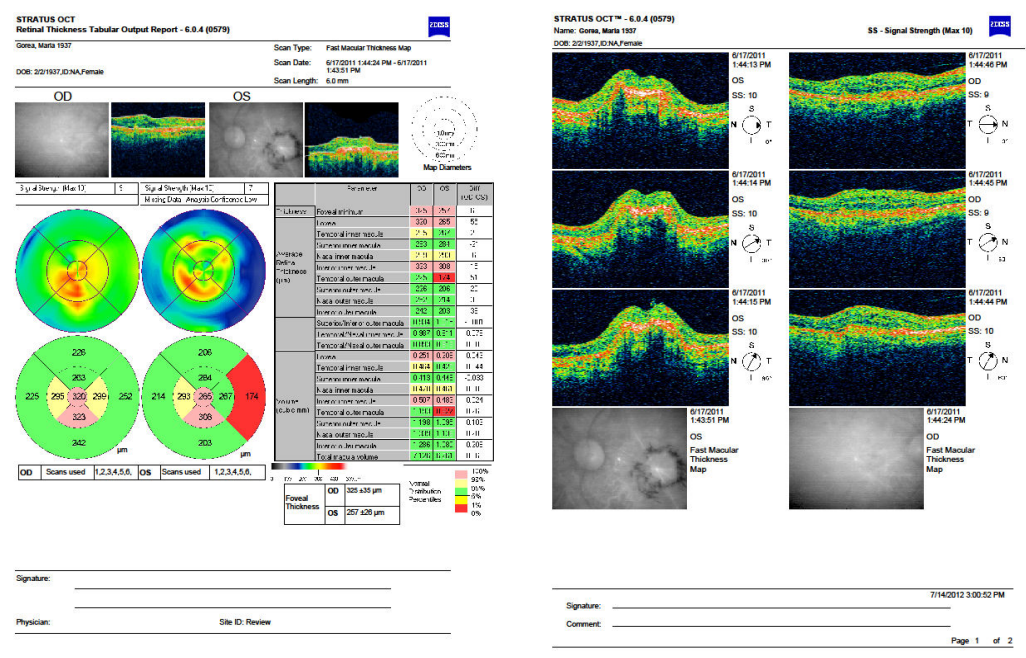

Figure 2. $a$ and b: Patient with bilateral wet AMD before Bevacizumab injections (courtesy of dr. H. Demea, Review Centre, Cluj-Napoca, Romania)
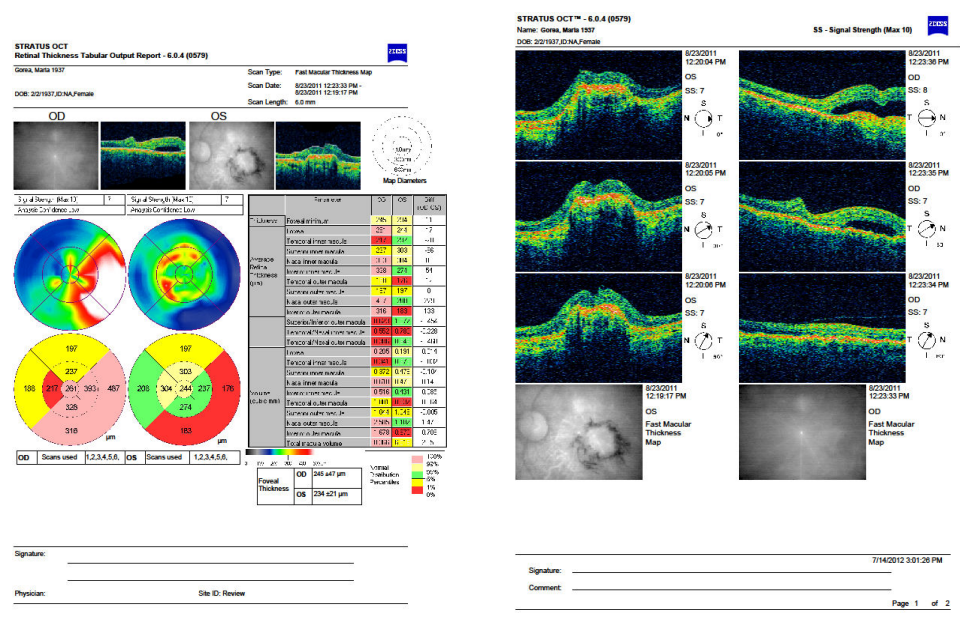

Figure 3. $\mathrm{a}$ and b: The case depicted in figure 2, after two intravitreal injections with Bevacizumab (courtesy of dr. H. Demea, Review Centre, Cluj-Napoca, Romania) 

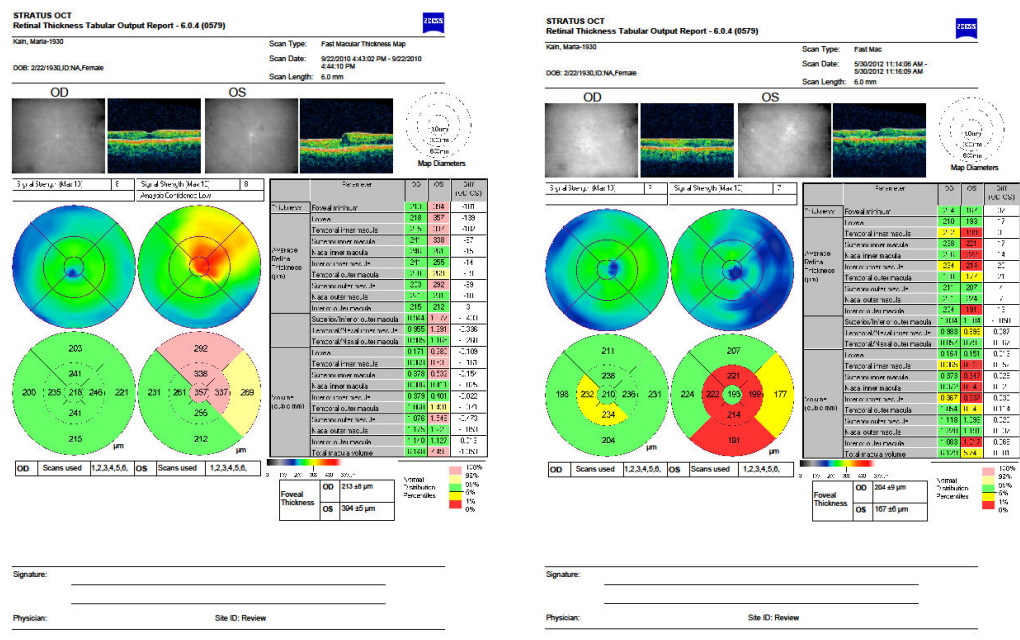

Figure 4. a: Macular edema in AMD (left eye) b: The same patient 20 months after Avastin
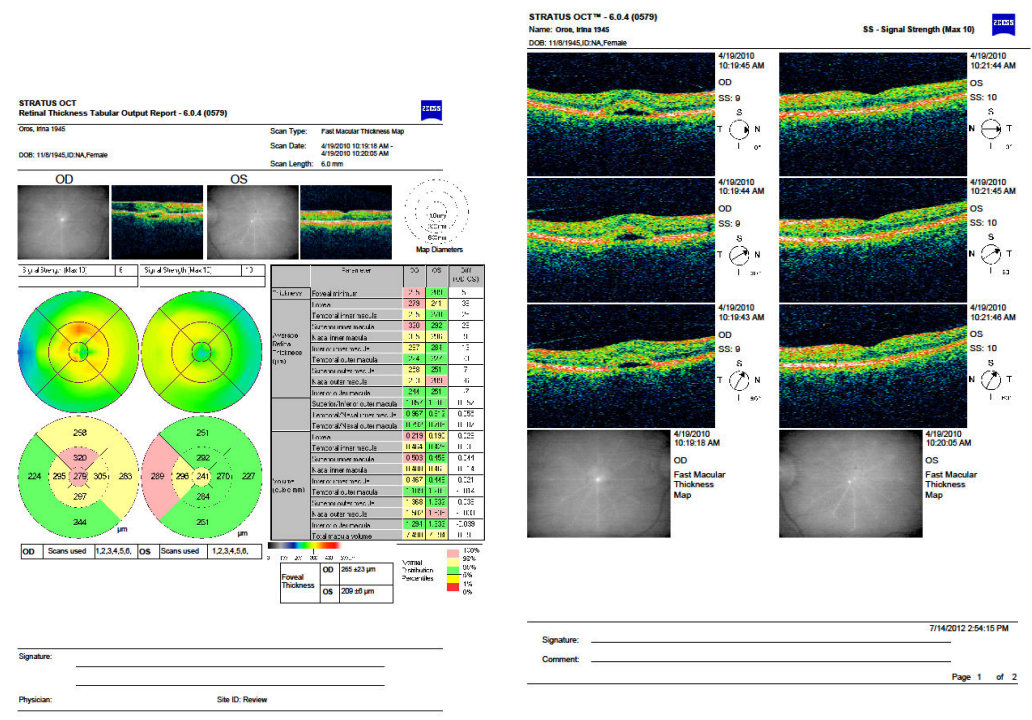

Figure 5. $a$ and b: RPE elevation in AMD patient before Avastin injection (courtesy of dr. H. Demea, Review Centre, Cluj-Napoca, Romania) 

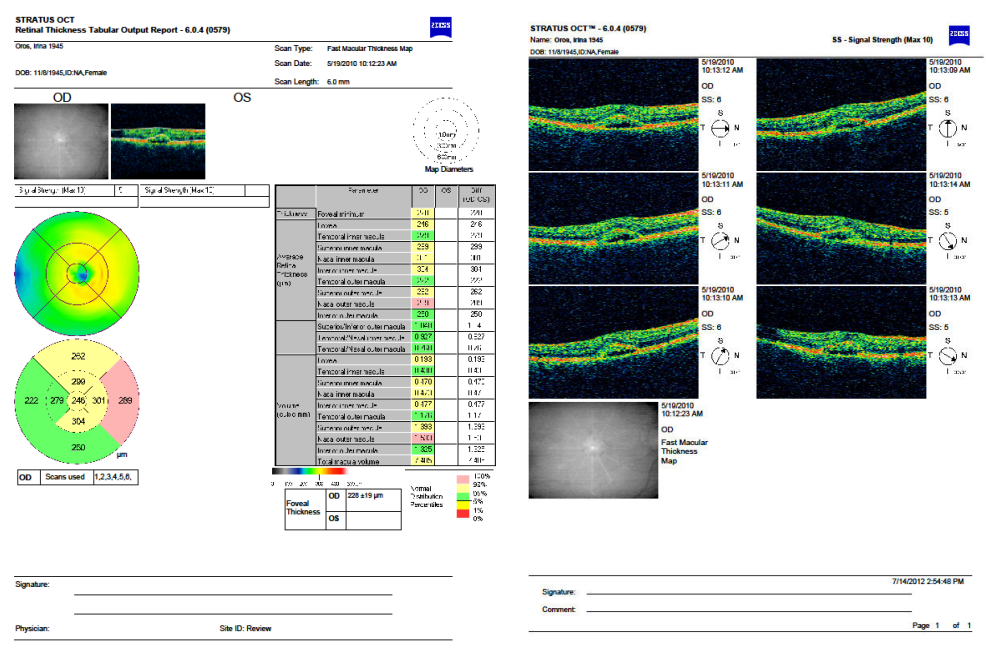

Figure 6. $a$ and b: The case depicted in figure 5, one month after Avastin injection (courtesy of dr. H. Demea, Review Centre, Cluj-Napoca, Romania)
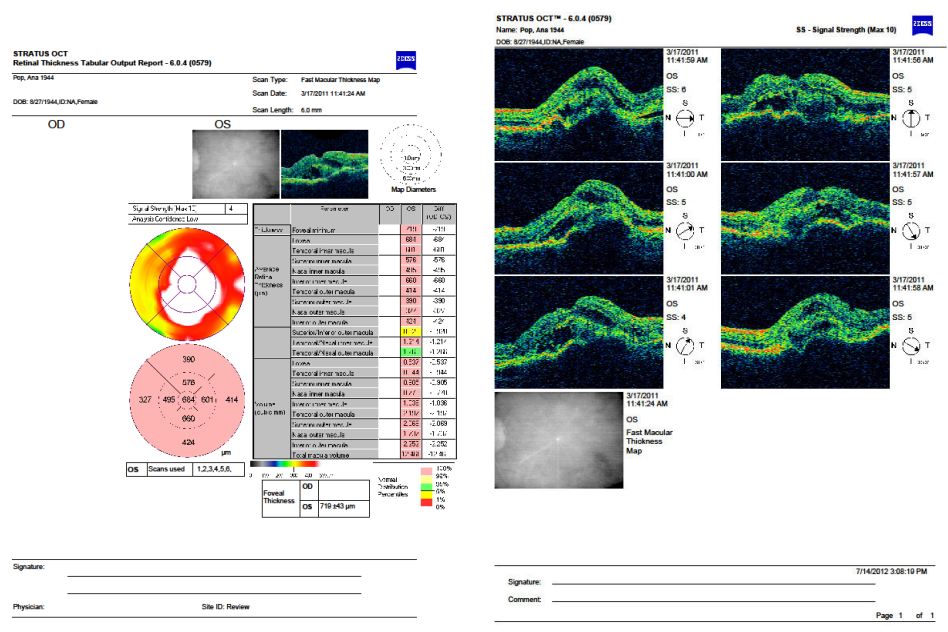

Figure 7. $a$ and b: Advanced case of AMD before Bevacizumab injection 


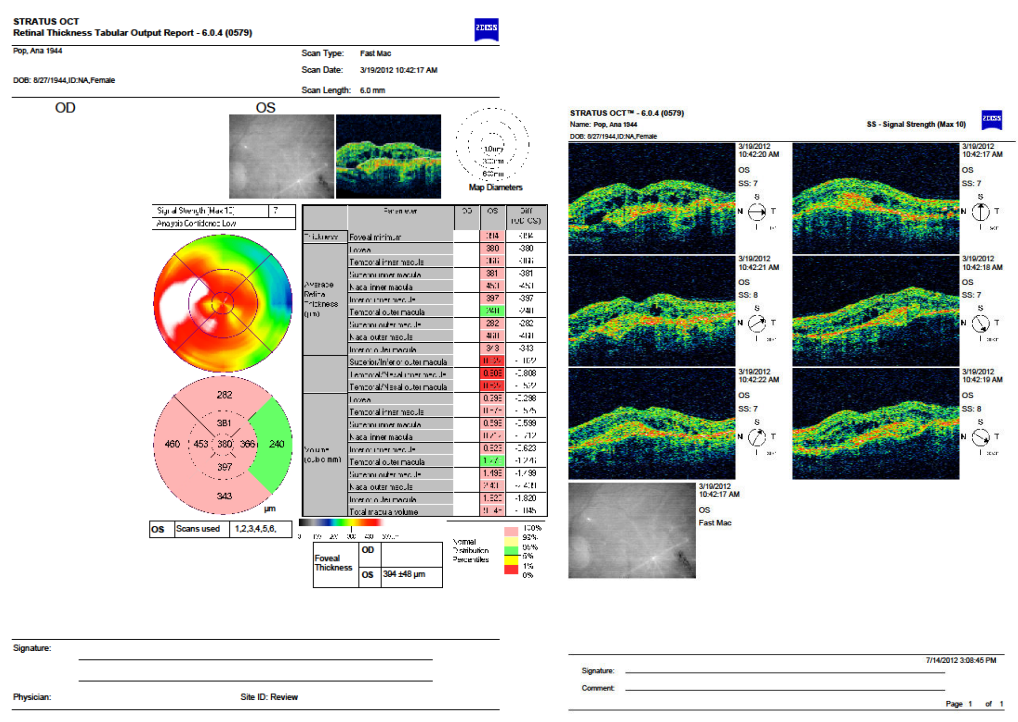

Figure 8. $a$ and b: The same case depicted in figure 7, one year after Avastin injections

\section{Cirrus OCT in clinical practice}

Average macular thickness is generated automatically with Cirrus-OCT. Each image had a 5 $\mu \mathrm{m}$ axial and $10 \mu \mathrm{m}$ transverse resolutions in tissue and consisted of either $512 \times 128$ volume cube or $200 \times 200$ volume cube with a maximum scan velocity of 27,000 axial scans per second. Cirrus OCT presents high resolution raster scanning capabilities.

Figure 9a presents the HD 5 line raster of a normal retina: the highly reflective retinal layers are red, the layers with intermediate reflectivity are green and the low reflectivity is translated intro blue color. The choriocapillaris under the RPE can also be seen. In figure $9 \mathrm{~b}$ the macular thickness map and the $512 \times 128$ volume cube are illustrated.

Figure 10a: irregularities of the RPE band which is elevated by a moderately reflective structure. Behind the RPE band: thick hyperreflective structure. Figure 10b: elevations of the RPE by hyperreflective structures.

Figure 11 illustrates the same patient as in figure 4, with Stratus OCT device. On figure 11a more details are shown, the RPE appears irregular, cysts in the neural retina with a hyporeflective content (fluid) are displayed, not visible on Stratus OCT. Figure 11b presents the macular thickness which is higher than with Stratus OCT. The difference comes from the different boundaries used by the algorihms of the two devices when measuring the macular thickness. The Cirrus OCT program measures the retinal thickness between the nerve fiber layer and the outer band of the RPE. The Stratus OCT program measures between the nerve 
fiber layer and the inner boundary of the RPE complex, though it has been reported that Stratus OCT has two outer reference lines: one at the junction between the inner/outer segment of the photoreceptor cells and the other at the inner boundary of the RPE. In consequence, the Cirrus outer reference band is deeper than the first mentioned Stratus external band and is closer to the second mentioned one. The correlation of thickness measurements between the two devices is modest, the Cirrus OCT provides greater measurement depth.

Figure 12: irregularities and thinning of the RPE band especially in the right eye, fluid in the retina, increased macular thickness.

Figure 13: RPE band appears thinned and elevated by a moderately reflective tissue: fluid, $\mathrm{CNV}$, increased central macular thickness. 3D macular cube

Figure 14: comparative aspects of the two eyes of the same patient: in the Right Eye the RPE band appears irregular and there is some fluid in the retina.

Figure 15: macular cube 200x200 shows increased central macular thickness, elevations of the RPE band, associated with increased vitreo-macular adhesion revealed by the 3D presentation of the macular cube.
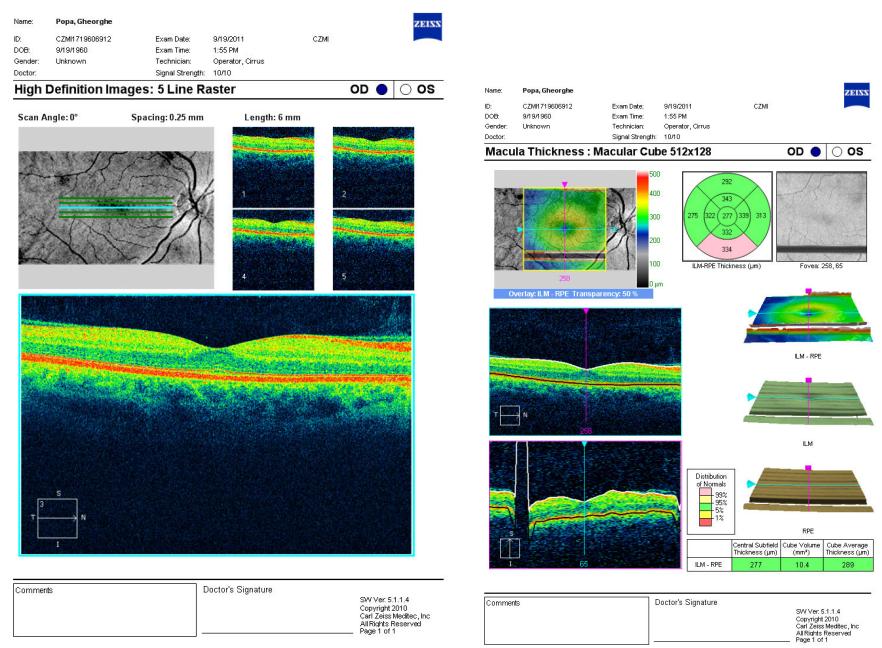

Figure 9. a: 5 line raster of a normal retina b: Normal macular thickness map 
(a)

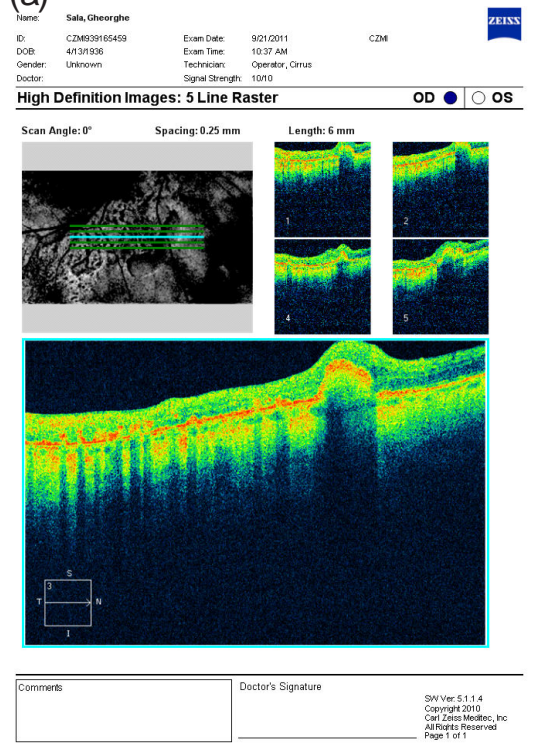

(b)

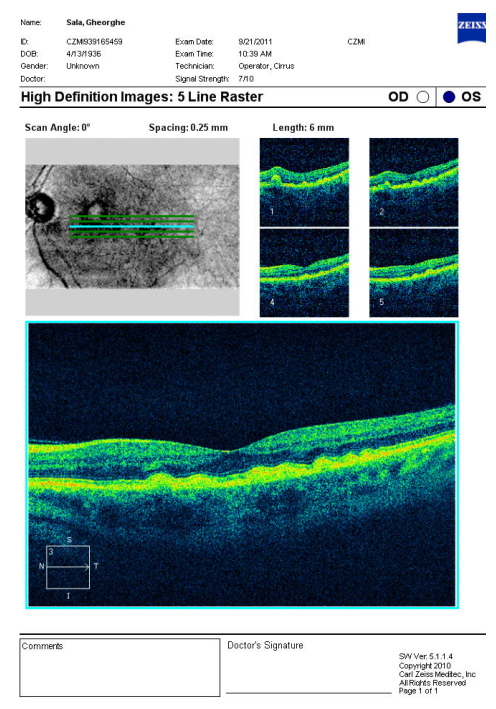

Figure 10. a: Irregularities of the RPE $b$ and $b$ : Elevations of the RPE band

(a)

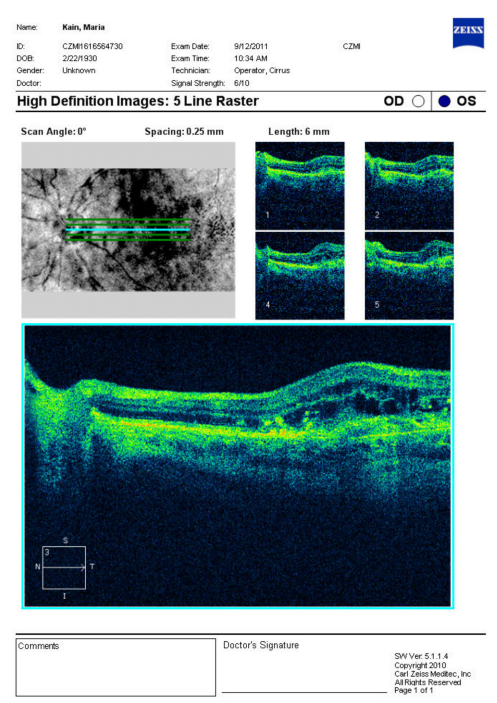

(b)
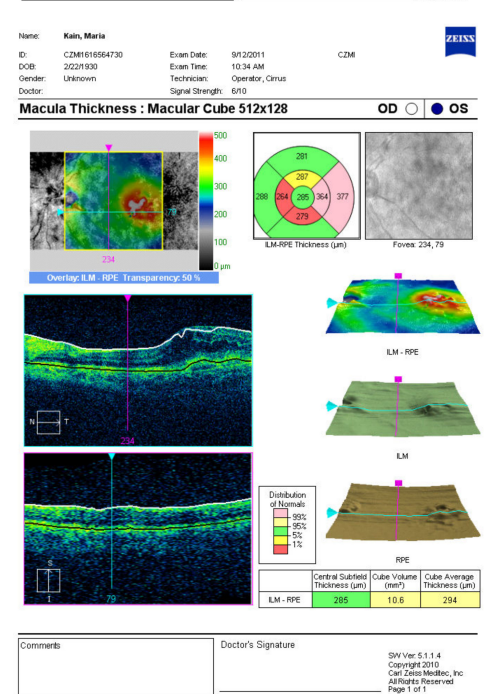

Figure 11. The same patient as in figure $4 \mathrm{a}$ and $\mathrm{b}$ 


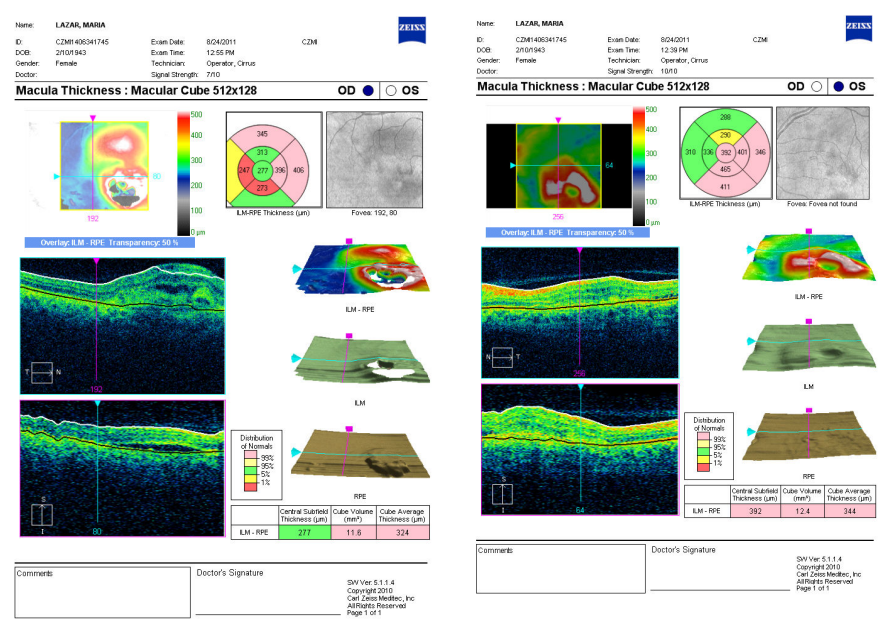

Figure 12. Increased macular thickness, RPE irregularities, fluid in the retina due to AMD
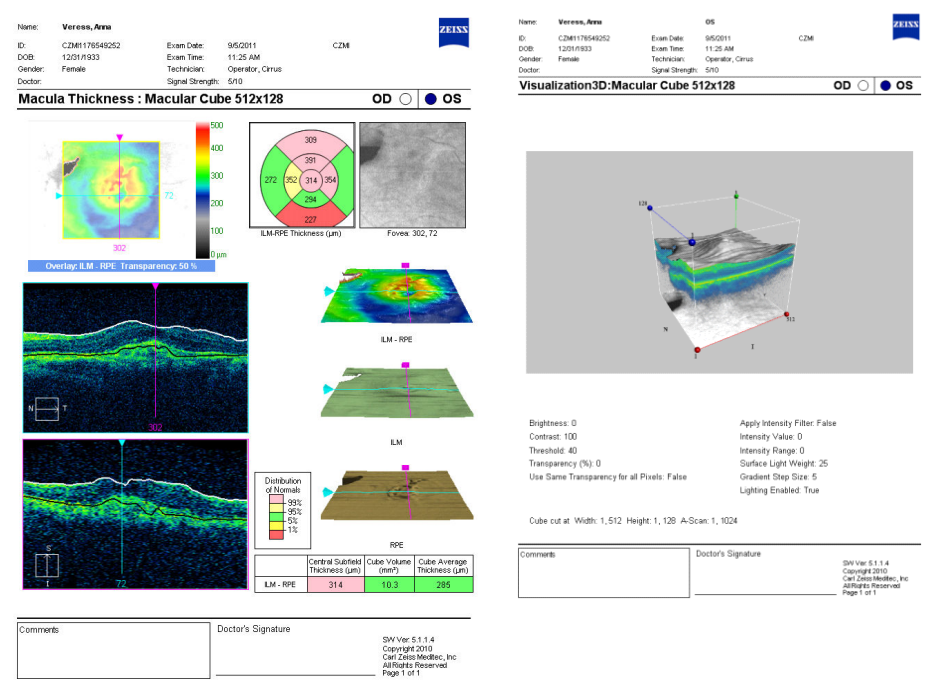

Figure 13. RPE band appears thinned and elevated by a moderately reflective tissue: fluid, CNV, increased central macular thickness. 3D macular cube 

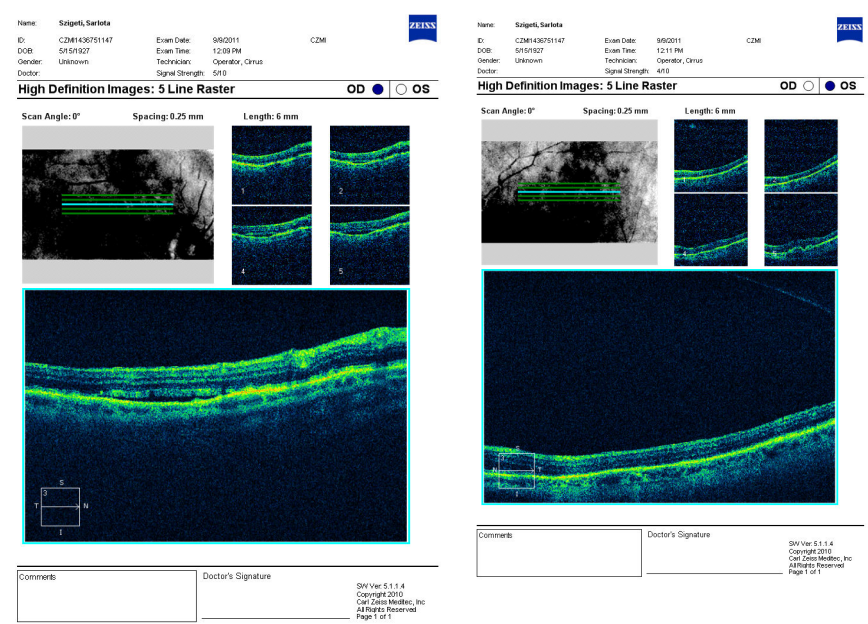

Figure 14. Comparative aspects of the two eyes of the same patient: in the Right Eye the RPE band appears irregular and there is some fluid in the retina.

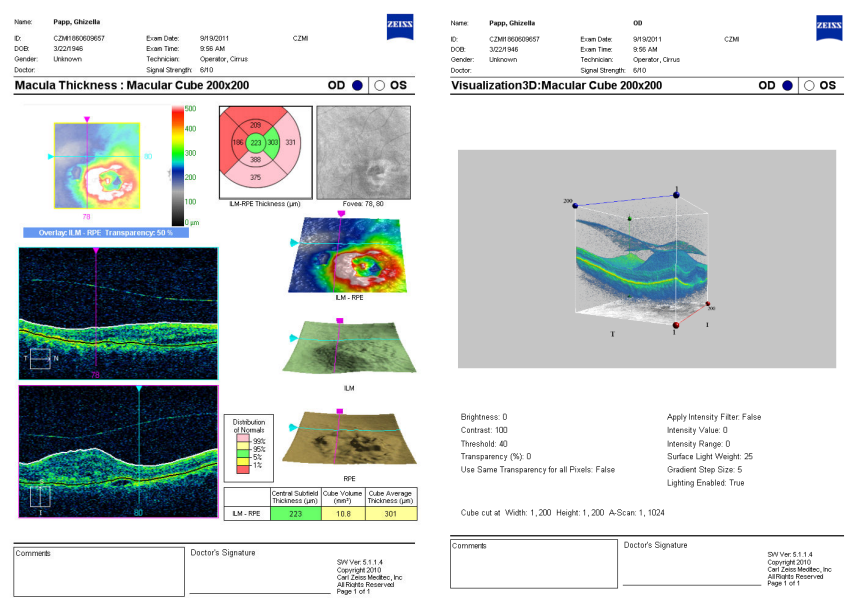

Figure 15. Macular cube 200×200 shows increased central macular thickness, elevations of the RPE band, associated with increased vitreo-macular adhesion revealed by the 3D presentation of the macular cube 


\section{Future directions}

A significant progress for neovascular AMD imaging was the development of FD-OCT technologies. They use a central wavelength of $800-850 \mathrm{~nm}$, a stationary reference arm, a high speed spectrometer and a charged-coupled device (CCD) line-scan camera. The mechanical scanning is not needed in order to detect light echoes simultaneously. As consequence, the aquisition speed increases to 25,000-52,000 A-scan/second. The axial resolution is of 3-7 $\mu \mathrm{m}$, significantly improving the signal-to-noise ratio and the detection of individual retinal layers and lesions components is possible [16].

\subsection{Limits of the current OCT examination techniques}

Despite the significant advantages previously mentioned, there are some limitations of SDOCT: motion and segmentation artifacts, interinstrument comparability [11,12]. Despite the significant progress in retinal imaging offered by the current OCT techniques, TD-OCT and SD-OCT have shortcomings originating in the limitation of resolution, both axial and lateral. The absorbtion of infrared radiation by the anterior segment structures and ocular media limits the image resolution. The axial resolution is limited by the image scattering by the ocular structures (so-called speckle noise) and the lateral resolution limitation is determined by the restricted numerical aperture of the optical system [16].

\subsection{Swept source OCT}

SS-OCT is another form of FD-OCT that uses a light source with the wavelength of approximately $1,050 \mathrm{~nm}$. A short cavity-swept laser replaces the superluminiscent diode laser. The emission has different frequencies that can be rapidly tuned over a broad bandwidth [11, 28]. A high speed complementary metal oxide semiconductor camera (CMOS) and two parallel photodetectors are used in order to obtain scan rates of $100,000-400,000$ A-scan/second, with the axial resolution of $5.3 \mu \mathrm{m}$ over a 4-mm imaging range.(trebuie modificat) [29]. Advantages: images to the level of individual photoreceptors are obtained, particularly when coupled with adaptive optics. With SS-OCT the socalled fringe washout (signals at the edges of the B-scan) is reduced as compared to SDOCT; the sensitivity is better with imaging depth; the image range is longer: approximately $7.5 \mathrm{~mm}$, which allows the evaluaiton of the anterior segment without the use of complex imaging techniques that might generate errors [9]; the efficiency of detection is higher; the dual balanced detection can be performed. The above mentioned advantages considerably decrease the patient-induced errors by movements and breathing and permit the better penetration in case of ocular media opacities [30]. Limits: even with best patient cooperation, images are still subject to artifacts. Therefore, various algorithms have been imagined in order to improve the resolution by eliminating these artifacts [30]. The potential application of OCT in the sub-RPE space and choroid is limited by its shallow penetration: approximately $1-3 \mathrm{~mm}$. The degree of choroidal penetration is determined by several factors: the proportion of scattered photons, the absorbtion spectrum of water, the scatter by the ocular media, the absorbtion by melanin [10]. Photon 
scattering is a phenomenon that influences the image formation in OCT: photons that are singly scattered add to the OCT signal, whereas photons that are scattered multiple times contribute to the background noise [31]. The large amount of water within the eye limits the light wavelengths that can be used [32]. The absorbtion spectrum of water has two regions where the light absorbtion is low: at approximately $950 \mathrm{~nm}$ and between $1,000-1,100 \mathrm{~nm}$ [30]. The devices with wavelenghts in the range of 1,000-1,100 $\mathrm{nm}$ can be used for the enhanced sub-RPE imaging, with ultrahigh-speed image aquisition and axial resolution in the range of $8 \mu \mathrm{m}$ [10]. This is useful in the managements of sub-RPE space diseases, particularly in AMD.

\subsection{Adaptive optics}

Adaptive optics correct ocular aberrations during image aquisition, making possible the obtaining of image resolution at the cellular level.

\subsection{Enhanced Depth Imaging OCT}

The principle of Enhanced depth imaging OCT (EDI-OCT) consists in placing the objective lens of the Spectralis SD-OCT device (Heidelberg Engineering) closer to the eye, with the obtaining of an inverted image, which allows the deeper structures to be placed closer to the zero delay with the subsequent better visualization of the choroid. This principle is combined with the high speed scanning, eye-tracking system, image-averaging technology, reduced noise and greater coverage of the macula. All these improvements lead to the possibility to create high resolution, repeatable and reliable images of the choroid [33].

\section{Conclusion}

At the present moment, OCT offers the most valuable data on the retinal structure. AMD is the retinal disease that benefited the most from the development of OCT techniques, especially the wet form of this disease. SD-OCT has superior depth resolution as compared to TD-OCT: currently, the axial resolution varies from $3-7 \mu \mathrm{m}$, depending on the SD-OCT model. OCT is more reliable than biomicroscopy in assessing the macular thickness and small neurosensory and RPE detachments. OCT is more reliable than FA in identifying the intraretinal and subretinal fluid, as on FA the fluid in the inner retina can mask the fluid in the outer retina. OCT is less precise in evaluating the geographic extent of fluid within the macula, as compared to FA. Due to the cross-sectional imaging, OCT allows the localization of a CNV in relationship with the RPE and the neurosensory retina. OCT is superior to FA in identifying CNV membranes that are obscured by pooling of dye or by tiny retinal haemorrhages. Cystoid macular edema is strongly associated with the classic CNV. OCT is very useful in AMD monitoring and decision-making after treatment: if fluid persists, re-treatment is indicated. 


\section{Author details}

Simona-Delia Ţălu

Address all correspondence to: simonatalu@yahoo.com

Department of Surgical Sciences and Medical Imaging, Ophthalmology, „Iuliu Haţieganu” University of Medicine and Pharmacy, Cluj-Napoca, Romania

\section{References}

[1] Lujan B J. Revealing Henle's Fiber Layer Using Spectral-domain OCT. Retin Physician 2011;8(1) 16-17.

[2] Lim LS, Mitchell P, Seddon JM et al. Age-related macular degeneration. Lancet 2012; 379: $1728-38$.

[3] El Maftouhi Q, Wolff B, Faysse MM. Kystes rétiniens externes dans la dégénérescence maculaire liée à l'âge exsudative : un nouvel aspect de l'OCT. J Fr d'Ophtalmol. $2010 ; 33$ : 605-609 doi:10.1016/j.jfo.2010.09.015.

[4] Talu SD, Talu S, Use of OCT Imaging in the Diagnosis and Monitoring of Age Related Macular Degeneration, in Age Related Macular Degeneration.The recent advances in basic research and clinical care, ed. Gui-Shuang Ying, Rijeka, Intech, 2012.

[5] Huang D, Swanson EA, Lin CP et al. Optical coherence tomography. Science 1991;22 1178-1181.

[6] Hee MR, Puliafito CA, Wong C et al. Quantitative assessment of macular edema with optical coherence tomography. Arch Ophthalmol. 1995;113 1019 - 1029.

[7] Ghazi N, Kirk T, Allam S et al. Quantification of Error in Optical CoherenceTomography Central Macular Thickness Measurement in Wet Age-related Macular Degeneration. Am J Ophthalmol 2009;148:90-96 doi:10.1016/j.ajo.2009.02.017

[8] F. G.Holz R. F. Spaide. Essentials in ophthalmology. Medical Retina, Berlin Heidelberg, 2005

[9] Costa RA, Skaf M, Melo LA Jr et al. Retinal assessment using optical coherence tomography. Prog Retin Eye Res. 2006;25 325-353.

[10] Keane PA, Ruiz-Garcia H, Sadda SR. Clinical applications of long-wavelength $(1,000-$ $\mathrm{nm}$ ) optical coherence tomography. Ophthalmic Surg Lasers Imaging 2011;42 S67S74. 
[11] Potsaid B, Baumann B, Huang D et al. Ultrahigh speed 1050nm speedswept source/ Fourier domain OCT retinal and anterior segment imaging at 100,000 to 400,000 axial scans per second. Opt Express. 2010;18 20029-20048

[12] Sull AC, Vuong LN, Price LL et al. Comparison of spectral/Fourier domain optical coherence tomography instruments for assessment of of normal macular thickness. Retina 2010;30 235-245.

[13] Menke M, Lala C, Framme C, Wolf S. The Ever-Evolving Role of Imaging in DME Management. Retin Physician 2012;9(4) 24-32.

[14] Lumbroso B, Rippoli M. Guide to Interpreting Spectral Domain Optical Coherence Tomography. James Allyn, Inc.; Dublin, CA; 2010

[15] Kiernan DF, Mieler WF, Hariprasad SM. Spectral-Domain Optical Coherence Tomography: A Comparison of Modern High-Resolution Retinal Imaging Systems Am J Ophthalmol 2010;149:18-31 doi:10.1016/j.ajo.2009.08.037

[16] Wojkowski M, Kaluzny B, ZawadziRJ. New directions in ophthalmic optical coherence tomography. Optom Vis Sci 2012 Mar 22 (Epub ahead of print).

[17] Murakami T, Nishijima K, Akagi T et al. Optical coherence tomographic reflectivity of photoreceptors beneath cystoid spaces in diabetic macular edema. Invest Ophthalmol Vis Sci 2012 Feb 8 (epub ahead of print)

[18] Salam A, Framme C, Wolf S. How SD-OCT is changing our view of DME. Retin Physician 2010;7(8) 41-46.

[19] Kiernan DF, Hariprasad HM, Chin EK et al. Prospective Comparison of Cirrus and Stratus Optical Coherence Tomography for Quantifying Retinal Thickness. Am J Ophthalmol. 2009;147 267-275 doi:10.1016/j.ajo.2008.08.018

[20] Philip L. Penfold, Jan M. Provis. Macular Degeneration. Springer, Berlin Heidelberg, 2005.

[21] Sulzbacher F, Kiss C, Munk M et al. Diagnostic Evaluation of Type 2 (Classic) Choroidal Neovascularization: Optical Coherence Tomography, Indocyanine Green Angiography, and Fluorescein Angiography. Am J Ophthalmol. 2011;152:799-806 doi: 10.1016/j.ajo.2011.04.011

[22] Spaide RF. Enhanced Depth Imaging Optical Coherence Tomography of Retinal Pigment Epithelial Detachment in Age-related Macular Degeneration. Am J Ophthalmol 2009;147:644-652 doi:10.1016/j.ajo.2008.10.005

[23] Penha FM, Rosenfeld PJ, Gregori G et al. Quantitative Imaging of Retinal Pigment Epithelial Detachments Using Spectral-Domain Optical Coherence Tomography. Am J Ophthalmol 2012;153:515-523 doi:10.1016/j.ajo.2011.08.031

[24] Mojana F, Cheng L, Bartsch DUG et al. The Role of Abnormal Vitreomacular Adhesion in Age-related Macular Degeneration: Spectral Optical Coherence Tomography 
and Surgical Results. Am J Ophthalmol. 2008;146: 218-227 doi:10.1016/j.ajo. 2008.04.027

[25] Lee SJ, Lee CS, Koh HJ. Posterior Vitreomacular Adhesion and Risk of Exudative Age-related Macular Degeneration: Paired Eye Study. Am J Ophthalmol. 2009; 147: 621-626, doi:10.1016/j.ajo.2008.10.003

[26] Krebs I, Brannath W, Glittenberg C et al. Posterior Vitreomacular Adhesion: A Potential Risk Factor for Exudative Age-related Macular Degeneration? Am J OPhthalmol. 2007; 144: 741-746 doi:10.1016/j.ajo.2007.07.024

[27] Manjunath V, Goren J, Fujimoto FJ et al. Analysis of Choroidal Thickness in Age-Related Macular Degeneration Using Spectral-Domain Optical Coherence Tomography. Am J Ophthalmol 2011;152: 663-668 doi:10.1016/j.ajo.2011.03.008

[28] Keane PA. Sadda SR. Predicting visual outcomes for macular disease using optical coherence tomography. Saudi Journal of Ophthalmology. 2011;25 145-158 doi: 10.1016/j.sjopt.2011.01.003

[29] Regatieri CV, Branchini L, Duker JS. The role of spectral-domain OCT in the diagnosis and management of neovascular age-related macular degeneration. Ophthalmic Sur Lasers Imaging. 201142 S56-S66.

[30] Raiji V, Walsh A, Sadda S. Future Directions in Retinal Optical Coherence Tomography. Retin Physician 2012;9(4) 33-37.

[31] Sharma U, Chang EW, Yun SH. Long-wavelength optical coherence tomography at 1.7 microm for enhanced imaging depth. Opt Express. 2008; 16 19712-19723.

[32] Unterhuber A, Povazay B, Hermann B, Sattmann H, Chavez-Pirson A, Drexler W. In vivo retinal optical coherence tomography at $1040 \mathrm{~nm}$; enhanced penetration into the choroid. Opt Express. 2005; 13 3252-3258.

[33] Spaide RF, Koisumi H, Posonni MC. Enhanced depth imaging spectral-domain optical coherence tomography. Am J Ophthalmol. 2008; 146 496-500. 
